\title{
Síndrome de burnout y la satisfacción laboral en profesionales de la salud
}

\author{
Rosario Margarita Yslado Méndez* 1,a; Roger Pedro Norabuena Figueroa ${ }^{1}$; Telmo Pablo Loli Poma ${ }^{1}$; Eva Zarzosa Marquez ${ }^{1}$; \\ Laura Padilla Castro ${ }^{2}$; Irene Pinto Flores ${ }^{3}$; Angelita Rojas Gamboa ${ }^{4}$
}

\section{RESUMEN}

Objetivo: Determinar las relaciones funcionales entre el burnout y la satisfacción laboral de los profesionales de la salud de dos hospitales del Perú.

Materiales y métodos: Estudio cuantitativo, observacional, correlacional de corte transversal. De una población de 620 profesionales de la salud se encuestaron a 177 profesionales (médicos, odontólogos, químicos farmacéuticos, psicólogos, enfermeros, obstetras, tecnólogos médicos, nutricionistas y trabajadores sociales). Los instrumentos de medición utilizados fueron el inventario de burnout de Maslach (MBI) y la escala de satisfacción laboral (SL-SPC), cuyas propiedades psicométricas fueron adecuadas.

Resultados: La tasa de prevalencia (TP) para el burnout alto fue 33,3\%. Mediante el análisis de ecuaciones estructurales, se encontró que existe relación significativa $(A G F I=0,946)$ y negativa (coeficiente estructural $=-0,62$ ) entre el burnout y la satisfacción laboral en los profesionales de la salud. La dimensión predominante del burnout fue la despersonalización $(37 \%=0,72 /(0,56+0,72+0,65)$; y el factor predominante de la satisfacción laboral fueron los beneficios económicos $(35 \%=0,99 /$ $(0,67+0,99+0,48+0,66)$.

Conclusiones: Existe relación funcional significativa y negativa entre el síndrome de burnout y la satisfacción laboral; el burnout es predictor de la satisfacción laboral. No se han encontrado diferencias significativas al comparar la tasa de prevalencia del burnout alto, según las variables sociodemográficas y laborales.

Palabras clave: Agotamiento psicológico; Satisfacción laboral; Grupos profesionales (Fuente: DeCS BIREME).

\section{Burnout syndrome and job satisfaction in health professionals}

\section{ABSTRACT}

Objective: To estimate the functional relationships between burnout and job satisfaction in health professionals of two hospitals in Peru.

Materials and methods: A quantitative, observational, correlational and cross-sectional research was carried out. Out of a study population of 620 health professionals, 177 (physicians, odontologists, pharmacists, psychologists, nurses, obstetricians, medical technologists, nutritionists and social workers) were interviewed. The Maslach Burnout Inventory $(\mathrm{MBI})$ and the Job Satisfaction Scale (SL-SPC) were used as measuring instruments, showing adequate psychometric properties.

Results: The high-burnout prevalence rate (TP) was $33.3 \%$. Using a structural equation analysis, a significant (AGFI = 0.946 ) and negative (structural coefficient $=-0.62$ ) relationship was found between burnout and job satisfaction in health professionals. The predominant dimension of burnout was depersonalization $(37 \%=0.72 /(0.56+0.72+0.65))$ and the predominant factor of job satisfaction was profits $(35 \%=0.99 /(0.67+0.99+0.48+0.66))$.

Conclusions: There is a significant and negative functional relationship between burnout syndrome and job satisfaction. Burnout is a predictor of job satisfaction. No significant differences were found when comparing the high-burnout prevalence rate regarding the sociodemographic and work-related variables.

Keywords: Burnout, psychological; Job satisfaction; Occupational groups (Source: MeSH NLM).

1. Universidad Nacional Santiago Antúnez de Mayolo. Ancash, Perú.

2. Universidad Autónoma del Estado de Morelos. Morelos, México.

3. Universidad Nacional del Santa. Ancash, Perú.

4. Hospital Regional Eleazar Guzmán Barrón. Ancash, Perú.

a. Licenciada en Psicología, Magister en Psicología Clínica, Doctora en Educación Internacional.

${ }^{*}$ Autor corresponsal 


\section{INTRODUCCIÓN}

El síndrome de burnout (SB) es un problema de salud mental y ocupacional que se presenta, con mayor frecuencia, entre los profesionales de la salud en todo el mundo. Afecta la calidad de vida laboral y la calidad de atención a los usuarios, como lo demuestran estudios previos en la última década ${ }^{(1-3)}$. Sin embargo, la mayoría de investigaciones se han realizado en grupos de enfermeros, y son escasos los reportes correlacionales sobre el SB y la satisfacción laboral (SL) en muestras representativas de profesionales de la salud, cuyas funciones y responsabilidad laboral difieren según la profesión, estructura y organización laboral. Por ello, es necesario realizar estudios para confirmar o refutar las afirmaciones al respecto ${ }^{(4)}$.

En el Perú, en general, la prevalencia global del SB en los profesionales de la salud es $12,5 \%{ }^{(5)}$, aunque diferentes estudios nacionales presentan resultados heterogéneos ${ }^{(6,7)}$. En Ancash, específicamente, una investigación en personal sanitario de 10 hospitales reportó un porcentaje de burnout alto que oscila entre $19,6 \%$ a $27,6 \%{ }^{(8)}$.

Como el burnout, la SL es un componente importante de la calidad de atención en salud. Surge cuando el profesional considera que la recompensa recibida es adecuada o equiparable a su rendimiento en el trabajo. Contrariamente, cuando se perciben bajas remuneraciones, que no compensan la labor realizada ni satisfacen las necesidades, se genera insatisfacción laboral ${ }^{(9)}$.

Estudios previos en profesionales y trabajadores de la salud, se observa que existe menor SL a mayores niveles de burnout, lo que tiene un efecto significativo en la calidad de vida en el trabajo y en la calidad asistencial (10,11). Además, se encontró que los enfermeros peruanos con burnout bajo, perciben mayor SL. La Superintendencia Nacional de Salud de Perú, asigna importancia a la relación existente entre el SB y la SL, y mide periódicamente el SB de los profesionales de la salud del Perú, como parte de la evaluación de satisfacción laboral de este grupo ${ }^{(12)}$.

Por lo expuesto, es necesario estudiar las relaciones entre el burnout y la SL, para generar nuevos conocimientos que contribuyan con el bienestar psicológico de los profesionales de salud y el desarrollo organizacional de los hospitales investigados, lo que incide en la mejora de la calidad asistencial, eficiencia y productividad de los profesionales sanitarios, y en la calidad de vida de la comunidad beneficiaria ${ }^{(13)}$.

El objetivo general del estudio fue determinar las relaciones funcionales entre el SB y la SL, en profesionales de la salud.

\section{MATERIALES Y MÉTODOS}

Diseño del estudio

Estudio cuantitativo, observacional, correlacional y transversal, realizado en profesionales de la salud de dos hospitales del Ministerio de Salud (Minsa) de Chimbote (Ancash, Perú).

\section{Población y muestra}

La población de estudio estuvo conformada por 620 profesionales de la salud, distribuidos en dos hospitales del Minsa. El muestreo fue aleatorio, estratificado con afijación proporcional por hospitales, con un error del 5 $\%$ y un nivel de confianza de $95 \%$. La muestra ha sido seleccionada de acuerdo a criterios de inclusión (edad, sexo, condición laboral, experiencia laboral y participación voluntaria mediante consentimiento informado escrito) y de exclusión (estar recibiendo tratamiento psicológico o psiquiátrico y no estar laborando por algún tipo de licencia). La muestra total fue de 177 profesionales de la salud, que incluyó enfermeros (47\%), médicos (26\%), obstetras (12\%), nutricionistas $(3 \%)$, tecnólogos médicos $(5 \%)$, odontólogos (1\%), psicólogos (1\%), químico farmacéutico (1\%) y traba-jadores sociales.

\section{Variables y mediciones}

Las variables SB (variable independiente) y la SL (variable dependiente), se midieron bajo las mismas condiciones en los hospitales investigados, en el turno diurno y en el período de febrero-marzo de 2018. Se utilizó la encuesta y los instrumentos de medición fueron la ficha sociodemográfica laboral (para recoger información general sobre las características personales, sociodemográficas y laborales de los participantes), el inventario de burnout de Maslach $(\mathrm{MBI})$ o del síndrome del "quemado" por estrés laboral asistencial y la escala de satisfacción laboral (SLSPC) de Palma.

El MBI consta de 22 ítems con siete respuestas posibles de acuerdo a la escala de Lickert $(0=$ Nunca, $1=$ Pocas veces al año o menos, 2= Una vez al mes o menos, 3= Unas pocas veces al mes o menos, 4= Una vez a la semana, 5= Pocas veces a la semana y $6=$ Todos los días) ${ }^{(14)}$. Tiene validez de contenido (Lawshe $=1,00$ ), validez de constructo mediante el análisis factorial (prueba de esfericidad de Bartlett $\mathrm{X} 2=535,869$ ) y fiabilidad (alfa de Cronbach $=0,867$ ). Para este estudio los puntos de corte se determinaron del puntaje general del SB, como en otras investigaciones ${ }^{(15,16)}$ así, se consideraron niveles bajo ( 0 a 19 puntos), nivel medio (20 a 32), y alto (33 a 132 puntos). Los percentiles obtenidos de la muestra de estudio, fueron concordantes con los percentiles propuestos por Maslach y Jackson: bajo (centiles 1 a 33), medio (centiles 34 a 66) y alto (centiles 57 a 99$)^{(14)}$.

La SL-SPC de Palma consta de 27 ítems en una escala 
dimensional de alternativas ${ }^{(17)}$, con validez de contenido (Lawshe $=1,00$ ), validez de constructo mediante el análisis factorial (prueba de esfericidad de Bartlett $X^{2}=560,210$ ) y fiabilidad (alfa de Cronbach $=0,820$ ). La validez de contenido se ha obtenido mediante criterio de jueces expertos para los instrumentos de medición. La validez de constructo y fiabilidad se obtuvieron a través de un estudio piloto en los profesionales de la salud de ambos hospitales del Minsa, cuya muestra piloto no fue parte de la presente investigación.

\section{Análisis estadístico}

Los datos se procesaron con los programas estadísticos SPSS (versión 24,0 trial) y AMOS (versión 24,0 trial). Para relacionar la tasa de prevalencia y variables sociodemográficas-laborales, se usó el análisis estadístico inferencial, y se realizaron las pruebas $U$ de Mann Whitney (dos grupos independientes), y $\mathrm{H}$ de Kruskal Wallis (tres a más grupos independientes) para el caso no paramétrico, debido a que los puntajes del SB no se aproximan a una distribución normal (Kolmogórov-Smirnov=0,092; gl=177, sig=0,001). El análisis multivariado se realizó mediante la estimación del modelo de ecuaciones estructurales (MES), por medio del método de distribución libre asintótica (pues las variables analizadas no cumplieron el supuesto de normalidad multivariante), para identificar las relaciones estructurales en los profesionales de la salud de los hospitales investigados.

\section{Consideraciones éticas}

Se solicitó la autorización a los directores de los hospitales en los cuales se realizó el estudio y la firma del consentimiento informado de parte del profesional contactado. Se consignó un código para proteger la identidad del participante y así cumplir con criterios éticos de anonimato y confidencialidad ${ }^{(18)}$.

\section{RESULTADOS}

Para establecer el perfil epidemiológico, se siguió el modelo propuesto por Bonita, Beaglehole y Kjellstróm ${ }^{(19)}$ y se encontró un $33,3 \%$ de tasa de prevalencia (TP) para el SB alto. Se establecieron relaciones entre el SB alto y las variables sociodemográficas-laborales de sexo, edad, estado civil, profesión, condición laboral, tiempo de experiencia laboral y número de horas de contacto con los pacientes. Los resultados obtenidos mostraron que las características sociodemográficas y laborales no presentaron diferencias significativas en relación al SB; es decir, los resultados revelaron un comportamiento independiente entre el SB y las variables sociodemográficas. Sin embargo, se observó que los profesionales de la salud con SB alto, en mayoría fueron mujeres (35,1\%); entre 30 a 39 años (40,9\%); con estado civil divorciado $(57,0 \%)$; de profesión químico-farmacéutico y cirujano dentista (50,0 \%); en condición de nombrado (34,5\%); con 20 a 29 años de experiencia (38,3\%); y trabajaban 8 horas diarias en contacto con pacientes $(55,0 \%$ ) (Tabla 1$)$.

Tabla 1. Prevalencia del síndrome de burnout y las variables sociodemográficas-laborales en los profesionales de la salud

\begin{tabular}{|c|c|c|c|c|c|c|c|c|c|c|}
\hline \multirow{3}{*}{\multicolumn{2}{|c|}{ Variable }} & \multirow{2}{*}{\multicolumn{2}{|c|}{ Frecuencia }} & \multicolumn{6}{|c|}{ Síndrome de burnout } & \multirow{3}{*}{$\begin{array}{c}\text { Pruebas } \\
\text { estadísticas }\end{array}$} \\
\hline & & & & \multicolumn{2}{|c|}{ Bajo } & \multicolumn{2}{|c|}{ Medio } & \multicolumn{2}{|c|}{ Alto } & \\
\hline & & n & $\%$ & $n$ & TP & $\mathbf{n}$ & TP & n & TP & \\
\hline \multicolumn{2}{|c|}{ Prevalencia global } & --- & --- & 67 & 37,9 & 51 & 28,8 & 59 & 33,3 & --- \\
\hline \multirow{2}{*}{ Sexo } & Varón & 46 & $26,0 \%$ & 21 & 45,7 & 12 & 26,1 & 13 & 28,3 & \multirow{2}{*}{$p=0,092$} \\
\hline & Mujer & 131 & $74,0 \%$ & 46 & 35,1 & 39 & 29,8 & 46 & 35,1 & \\
\hline \multirow{4}{*}{$\begin{array}{l}\text { Grupos de } \\
\text { edad (años) }\end{array}$} & 20 a 29 & 6 & $3,4 \%$ & 3 & 50,0 & 2 & 33,3 & 1 & 16,7 & \multirow{4}{*}{$p=0,723$} \\
\hline & 30 a 39 & 44 & $24,9 \%$ & 15 & 34,1 & 11 & 25,0 & 18 & 40,9 & \\
\hline & 40 a 49 & 38 & $21,5 \%$ & 16 & 42,1 & 10 & 26,3 & 12 & 31,6 & \\
\hline & 50 a 60 & 89 & $50,3 \%$ & 33 & 37,1 & 28 & 31,5 & 28 & 31,5 & \\
\hline \multirow{7}{*}{ Estado civil } & Conviviente & 12 & $6,8 \%$ & 5 & 41,7 & 4 & 33,3 & 3 & 25,0 & \multirow{7}{*}{$p=0,841$} \\
\hline & Casado & 120 & $67,8 \%$ & 44 & 36,7 & 38 & 31,7 & 38 & 31,7 & \\
\hline & Divorciado & 7 & $4,0 \%$ & 3 & 42,9 & 0 & 0,0 & 4 & 57,1 & \\
\hline & Separado & 2 & $1,1 \%$ & 1 & 50,0 & 0 & 0,0 & 1 & 50,0 & \\
\hline & Viudo & 2 & $1,1 \%$ & 1 & 50,0 & 0 & 0,0 & 1 & 50,0 & \\
\hline & Soltero & 32 & $18,1 \%$ & 12 & 37,5 & 8 & 25,0 & 12 & 37,5 & \\
\hline & Otros & 2 & $1,1 \%$ & 1 & 50,0 & 1 & 50,0 & 0 & 0,0 & \\
\hline
\end{tabular}




\begin{tabular}{|c|c|c|c|c|c|c|c|c|c|c|}
\hline \multirow{2}{*}{\multicolumn{2}{|c|}{ Variable }} & \multirow{2}{*}{\multicolumn{2}{|c|}{ Frecuencia }} & \multicolumn{6}{|c|}{ Síndrome de burnout } & \multirow{3}{*}{$\begin{array}{c}\text { Pruebas } \\
\text { estadísticas }\end{array}$} \\
\hline & & & & \multicolumn{2}{|c|}{ Bajo } & \multicolumn{2}{|c|}{ Medio } & \multicolumn{2}{|c|}{ Alto } & \\
\hline & & $\mathbf{n}$ & $\%$ & $\mathbf{n}$ & TP & $\mathbf{n}$ & TP & $\mathbf{n}$ & TP & \\
\hline \multirow{9}{*}{ Profesión } & Lic. en enfermería & 84 & $47,5 \%$ & 25 & 29,8 & 32 & 38,1 & 27 & 32,1 & \multirow{9}{*}{$p=0,749$} \\
\hline & Médico & 46 & $26,0 \%$ & 21 & 45,7 & 9 & 19,6 & 16 & 34,8 & \\
\hline & Obstetra & 21 & $11,9 \%$ & 11 & 52,4 & 3 & 14,3 & 7 & 33,3 & \\
\hline & Nutricionista & 6 & $3,4 \%$ & 3 & 50,0 & 1 & 16,7 & 2 & 33,3 & \\
\hline & Tecnólogo médico & 9 & $5,1 \%$ & 3 & 33,3 & 3 & 33,3 & 3 & 33,3 & \\
\hline & Trabajador social & 5 & $2,8 \%$ & 2 & 40,0 & 1 & 20,0 & 2 & 40,0 & \\
\hline & Químico & 2 & $1,1 \%$ & 0 & 0,0 & 1 & 50,0 & 1 & 50,0 & \\
\hline & Cirujano dentista & 2 & $1,1 \%$ & 0 & 0,0 & 1 & 50,0 & 1 & 50,0 & \\
\hline & Psicólogo & 2 & $1,1 \%$ & 2 & 100,0 & 0 & 0,0 & 0 & 0,0 & \\
\hline \multirow{2}{*}{$\begin{array}{l}\text { Condición } \\
\text { laboral }\end{array}$} & Nombrado & 148 & $83,6 \%$ & 55 & 37,2 & 42 & 28,4 & 51 & 34,5 & \multirow{2}{*}{$p=0,522$} \\
\hline & Contratado & 29 & $16,4 \%$ & 12 & 41,4 & 9 & 31,0 & 8 & 27,6 & \\
\hline \multirow{4}{*}{$\begin{array}{c}\text { Años de } \\
\text { experiencia }\end{array}$} & 1 a 9 & 64 & $36,2 \%$ & 26 & 40,6 & 17 & 26,6 & 21 & 32,8 & \multirow{4}{*}{$p=0,107$} \\
\hline & 10 a 19 & 37 & $20,9 \%$ & 15 & 40,5 & 9 & 24,3 & 13 & 35,1 & \\
\hline & 20 a 29 & 47 & $26,6 \%$ & 11 & 23,4 & 18 & 38,3 & 18 & 38,3 & \\
\hline & 30 & 29 & $16,4 \%$ & 15 & 51,7 & 7 & 24,1 & 7 & 24,1 & \\
\hline \multirow{7}{*}{$\begin{array}{l}\text { Horas diarias } \\
\text { de contacto } \\
\text { con pacientes }\end{array}$} & 2 & 2 & $1,1 \%$ & 0 & 0,0 & 2 & 100,0 & 0 & 0,0 & \multirow{7}{*}{$p=0,677$} \\
\hline & 4 & 7 & $4,0 \%$ & 3 & 42,9 & 2 & 28,6 & 2 & 28,6 & \\
\hline & 5 & 8 & $4,5 \%$ & 3 & 37,5 & 3 & 37,5 & 2 & 25,0 & \\
\hline & 6 & 100 & $56,5 \%$ & 41 & 41,0 & 29 & 29,0 & 30 & 30,0 & \\
\hline & 7 & 2 & $1,1 \%$ & 0 & 0,0 & 2 & 100,0 & 0 & 0,0 & \\
\hline & 8 & 20 & $11,3 \%$ & 5 & 25,0 & 4 & 20,0 & 11 & 55,0 & \\
\hline & 12 & 38 & $21,5 \%$ & 15 & 39,5 & 9 & 23,7 & 14 & 36,8 & \\
\hline
\end{tabular}

p: significación calculada

Con respecto a la relación funcional entre los puntajes brutos del SB y la SL, se observa que el factor latente del SB influye de manera negativa en el factor latente de la SL $(-0,62)$; mientras que la despersonalización incide de manera negativa sobre la realización personal $(-0,34)$. Asimismo, los efectos indirectos de los beneficios económicos se encuentran en asociación covariada negativa con los efectos indirectos de la significación de la tarea $(-0,22)$. Según los indicadores de ajuste, se puede inferir que el modelo estructural estimado es aceptable, pues cumple al menos uno de los indicadores de ajuste (Índice ajuste comparativo: GFI = 0,979) ${ }^{(20)}$ (Figura 1).

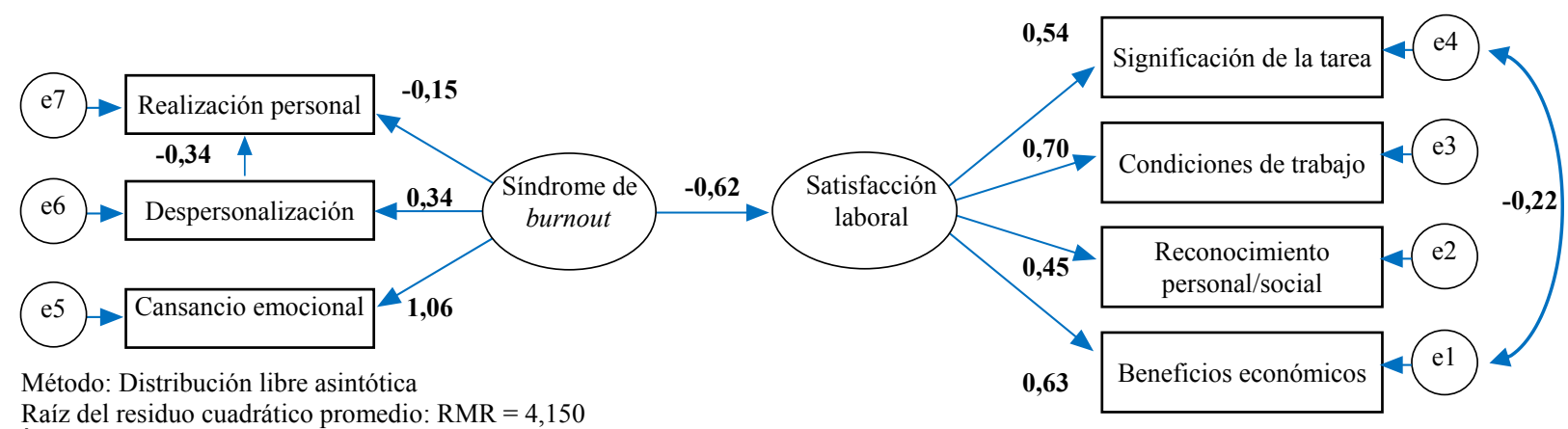

Raíz del residuo cuadrático promedio: $\mathrm{RMR}=4,150$

Índice de bondad de ajuste: $\mathrm{GFI}=0,979$

Índice de ajuste

Figura 1. Coeficientes estandarizados del MES del síndrome de burnout y la satisfacción laboral en los profesionales de la salud 
Asimismo, se encontró que la dimensión predominante del SB fue la dimensión despersonalización, por tener mayor coeficiente en valor absoluto $(0,72)$, y representa un 37 $\%(37 \%=0,72 /(0,56+0,72+0,65)$ de peso sobre el SB. Según los indicadores de ajuste, el modelo estructural estimado es aceptable, pues cumple al menos uno de los indicadores de ajuste (Índice de ajuste Normado $(\mathrm{NFI})=1$; Índice de ajuste comparativo $(\mathrm{GFI})=1^{(20)}($ Figura 2$)$.

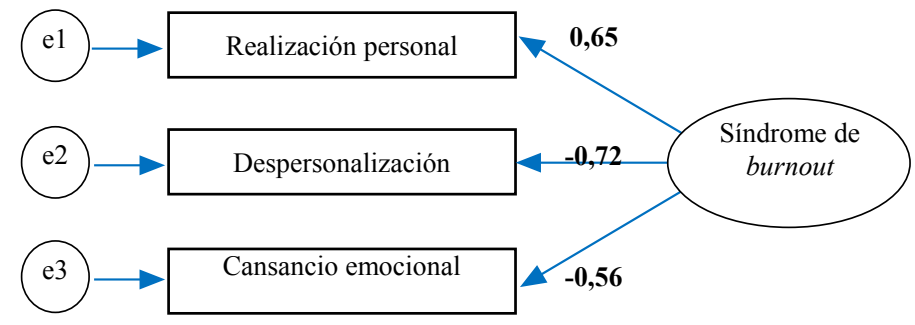

Método: Distribución libre asintótica

Raíz del residuo cuadrático promedio: $\mathrm{RMR}=0,000$

Índice de bondad de ajuste: $\mathrm{GFI}=1,000$

Índice de ajuste normalizado: NFI = 1,000

Figura 2. Coeficientes estandarizados del modelo estructural del síndrome de burnout en los profesionales de la salud

La dimensión predominante de la SL, fue el factor de beneficios económicos, por presentar mayor coeficiente $(0,99)$ y representar el $35 \%(35 \%=0,99 /$ $(0,67+0,99+0,48+0,66)$ de peso ejercido en la SL. Asimismo, la dimensión significación de la tarea afecta negativamente a los beneficios económicos. Además, las cuatro dimensiones inciden de manera directa sobre la SL de los profesionales de la salud. De acuerdo a los indicadores de ajuste, el modelo estructural estimado es aceptable, cumple al menos uno de los indicadores de ajuste :índice de ajuste normado $(\mathrm{NFI})=0,997$; índice de ajuste comparativo $(\mathrm{GFI})=1$; índice ajustado de bondad de ajuste $(\mathrm{AGFI})=0,995$ e índice relativo de ajuste $(\mathrm{RFI})=$ $0,980^{(20)}$ (Figura 3).

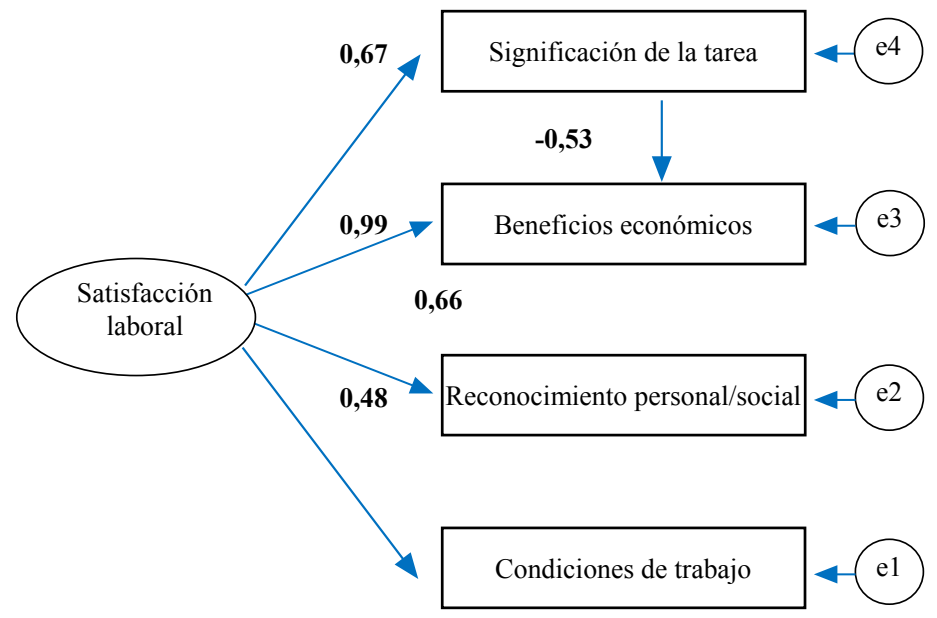

Método: Distribución libre asintótica

Raíz del residuo cuadrático promedio: $\mathrm{RMR}=0,155$

Índice de bondad de ajuste: $\mathrm{GFI}=1,000$

Índice de ajuste normalizado: NFI $=0,997$

Figura 3. Coeficientes estandarizados del modelo estructural de la satisfacción laboral en los profesionales de la salud 


\section{DISCUSIÓN}

El burnout es muy alto en trabajadores de la salud de Latinoamérica y España, oscilaría entre 30 a $40 \%$ (21), similar a la prevalencia para el SB alto encontrado en este estudio y que se presenta con mayor frecuencia en los siguientes casos: mujeres, entre los 30 y 39 años, en divorciados, químico-farmaceúticos y dentistas, nombrados, con experiencia laboral de 20 a 29 años y que trabajan 8 horas diarias en contacto con los pacientes. Sin embargo, las variables sociodemográficas y laborales descritas no tienen efecto significativo sobre el SB; es decir indistintamente cualquier profesional de la salud que labora en los hospitales investigados, puede verse afectado por SB alto. Otros estudios realizados sobre la relación entre el SB y las variables sociodemográficas en profesionales de la salud del ámbito internacional y nacional, reportaron que algunas de las variables estudiadas, no son significativas con respecto al SB ${ }^{(22-24)}$.

De acuerdo a los resultados las variables laborales observadas en los profesionales de la salud, estarían incidiendo en el SB alto, y podrían ser explicadas por el cambio frecuente de sus autoridades; el nombramiento de jefaturas como cargos de confianza; la sobrecarga laboral; los conflictos interpersonales; insuficientes estímulos al buen desempeño laboral; poca oportunidad de promoción y asenso; inconformidad con el salario bajo; el profesional percibe que su desempeño laboral realizado en condiciones de trabajo inadecuados, no es gratificado económicamente (por las autoridades de salud), y socialmente (por los pacientes).

Encontramos que existe relación significativa y negativa entre el SB y la SL, es decir a menor nivel de SB, mayor es la SL (niveles satisfecho y muy satisfecho) de los profesionales de la salud, entonces se infiere que el burnout es un predictor de la SL. El resultado señalado en parte se explicaría, porque la mayoría de la muestra del estudio estuvo conformada por personal de enfermería y médicos quienes, además de estar expuestos a las variables moderadoras laborales descritas en el párrafo precedente, están en contacto seis o más horas diarias con el dolor y la muerte de pacientes de baja, mediana o alta complejidad o gravedad, aunado a la desvalorización social de las profesiones sanitarias y bajas remuneraciones, todo lo que generaría burnout alto y menor satisfacción laboral. Los resultados coinciden con estudios precedentes realizados en muestras de enfermeros y médicos a nivel internacional, nacional y regional (25-30), y en estudios de revisión sistemática ${ }^{(31)}$ y metaanálisis ${ }^{(32)}$. Asimismo, los resultados hallados, indican que el SB incide en la SL y no al contrario como se reportó en otros estudios ${ }^{(27,33)}$, o que el burnout pueda coexistir con niveles altos de SL ${ }^{(34)}$.

Los resultados también muestran que la despersonalización incide de manera negativa sobre la realización personal; es decir, las actitudes negativas hacia el propio rol profesional, generan deterioro cognitivo consistente en la baja realización laboral, el profesional se percibe ineficaz en su trabajo e incapaz de mejorar ${ }^{(35)}$. La despersonalización bien puede ser un mecanismo para afrontar los estresores organizacionales; pero resta calidad a la atención y provoca insatisfacción en los pacientes y en los mismos profesionales sanitarios. En estudios previos de muestras similares, encontraron puntuaciones altas para las dimensiones cansancio emocional y despersonalización (36).

De acuerdo a los resultados obtenidos, la dimensión predominante del SB es la despersonalización (representa $37 \%$ de peso sobre el SB), que, como actitud disfuncional en el trabajo, es mayor en los profesionales expuestos a niveles medio o alto de contacto continuo con el sufrimiento o muerte de pacientes, y al impacto negativo del ambiente psicosocial (33). El trato deshumanizado, indiferencia, cinismo, distancia relacional (debido al endurecimiento afectivo) de los profesionales de salud hacia los pacientes, conllevaría a que los pacientes y los familiares de estos, maltraten física y psicológicamente (insultos, amenazas), a los profesionales sanitarios que los atienden, en su lugar de trabajo. Del resultado se infiere que la dimensión despersonalización, es el signo patognomónico del SB, prevalente en la muestra investigada y en el colectivo profesional de salud del Perú ${ }^{(12)}$. Se reporta un resultado similar en una revisión sistemática sobre burnout en profesionales de enfermería de urgencias ${ }^{(37)}$. Asimismo, los altos niveles de cansancio emocional (coeficiente estandarizado=0,56) y despersonalización (coeficiente estandarizado=0,72), encontrados en este estudio, revelan la gravedad del problema, pues afecta la salud ocupacional-mental y bienestar del profesional de la salud, como la calidad de atención percibida por los usuarios ${ }^{(28,38)}$. Hallamos que el factor predominante de la satisfacción laboral, son los beneficios económicos. Además, los efectos indirectos de estos se encuentran en asociación covariada negativa con los efectos indirectos de la significación de la tarea, es decir ,los profesionales de salud, perciben que su puesto es importante, pues atender, cuidar y salvar la vida de los enfermos en los hospitales tienen un impacto sustancial en el trabajo y sociedad; pero la disposición para realizar las tareas laborales, es compensado con remuneraciones e incentivos económicos bajos, que incidirían negativamente en otras atribuciones asociadas, como baja realización profesional, percepción de injusticia.

En Perú, en los estudios realizados en el colectivo profesional de salud, la SL está relacionada con mejoras económicas ${ }^{(39,40)}$. En Turquía se encontró que el bienestar económico se asocia significativamente con la SL en profesionales sanitarios ${ }^{(41)}$. En general, los profesionales 
de la salud que laboran en diversas entidades, si bien están motivados por y en su trabajo, manifiestan menor satisfacción con el salario en relación a otras variables externas; es decir a nivel mundial y nacional los factores externos o higiénicos, como la carencia de un salario aceptable potencian la insatisfacción del personal de una organización ${ }^{(42)}$.

El descontento con el salario se relaciona con la insatisfacción laboral, que es un factor común del colectivo profesional de salud latinoamericano, que tiene mayor presión y carga de trabajo constante y laboran en condiciones de trabajo inadecuados, con valoración económica y social desfavorable, los cuales afectan la calidad de vida de los profesionales de la salud (compromiso vocacional, desempeño laboral, salud física y mental, vida personal y familiar) y la calidad de relación con los pacientes y compañeros de trabajo; entonces el SB alto asociado a la insatisfacción laboral, generaría mayor rotación, intención de abandono y renuncia laboral, como mecanismos de protección contra la insatisfacción $(27,29,43,44)$

Cabe indicar que la satisfacción laboral no depende solamente de los niveles salariales, sino del significado que el profesional da a sus tareas laborales. El trabajo puede ser causa de felicidad y bienestar de una persona, o todo lo contrario. La satisfacción profesional puede depender de muchos factores como congruencia con los valores personales, grado de responsabilidad, sentido del éxito profesional, niveles de aspiración, grados de libertad que procura el trabajo, entre otros. Cuanto más elevada sea la calidad de vida profesional, más satisfacciones procurará al empleado y se reducirá el grado de ausentismo. Por otra parte, unos buscan en el trabajo reconocimiento o implicación y otros, socialización y estímulo; por lo tanto, es pertinente implementar programas de mejora, para que los empleados sientan mayor satisfacción y asignen importancia a su quehacer laboral ${ }^{(45)}$.

Según los resultados, además de los beneficios económicos y significación de la tarea, otros factores incidirían directamente, pero con menor fuerza, en los niveles de SL, por ejemplo, condiciones de trabajo y reconocimiento personal, social o ambos. También habría otros elementos distintos a los evaluados, que afectarían la SL, como los indicadores del significado de la tareas (competencias, identidad de la tarea, autonomía) ${ }^{(46)}$, los factores individuales y organizacionales como el apoyo socioemocional del supervisor y los compañeros de trabajo, el reto del trabajo, el sistema de recompensas justas, condiciones favorables del trabajo, compatibilidad entre personalidad y puesto de trabajo ${ }^{(47)}$, el clima laboral y el compromiso laboral, que se correlacionan positivamente con la mejora de la calidad de atención percibida por los pacientes y la reducción de intención de rotación ${ }^{(43,48)}$.
Una limitación del estudio fue que, al realizar la encuesta, no todos los profesionales aceptaron participar con consentimiento informado escrito, otros estuvieron de licencia por diferentes motivos. Entonces, algunos profesionales con mayores o menores niveles de SB o SL a lo encontrado en el estudio, no participaron en esta investigación; sin embargo, como la población fue amplia se logró completar la muestra de estudio. Asimismo, los estudios precedentes se han realizado en condiciones heterogéneas con respecto a la calidad metodológica, los instrumentos de medición (originales o adaptados con diferentes propiedades psicométricas y puntos de corte, uso de diversos baremos para la población general o específicos para poblaciones investigados), los métodos estadísticos, la muestra (enfermeros o médicos), los entornos laborales (tipo de establecimiento de salud), y el lugar de investigación (ciudad, país), cuyos resultados resultó complejo comparar; por ende para contrastar los resultados se han considerado, preferentemente, las investigaciones realizadas con muestras de profesionales de la salud, procedentes de hospitales y que utilizaron como instrumento el $\mathrm{MBI}$, para la medición del SB.

Entre las fortalezas de este estudio tenemos, en primer lugar, los resultados que permitirán corroborar las aportaciones de estudios previos, que fortalecen los conocimientos sobre la relación entre el SB y la SL para mejorar la práctica profesional y las políticas del sector salud desde la gestión de recursos humanos, lo cual redundará en una mejor atención a los usuarios. En segundo lugar, el estudio confirma la estructura trifactorial adecuada del $\mathrm{MBI}$, como la validez y fiabilidad del modelo de ecuaciones estructurales para analizar la relación entre el SB y la SL y, finalmente, los resultados obtenidos mediante el modelo señalado son concluyentes, válidos y pueden ser generalizados a la población de estudio, por cuanto que la muestra ha sido representativa y los instrumentos con propiedades psicométricas adecuadas; por lo tanto la pertinencia y relevancia del estudio, será el sustento para el diseño de un programa de intervención ocupacional y organizacional.

Proponemos investigar acerca de los aspectos teóricos y psicométricos de la escala SL-SPC, para identificar factores desconocidos que inciden en esta escala y que deben reconocerse e incorporarse y lograr así una óptima medición del constructo citado. Asimismo, realizar estudios de perspectiva, para confirmar relaciones causaefecto entre el SB y la SL, y trabajos sobre las variables moderadoras y mediadoras, que explican indirectamente 0 median en la relación entre el SB y la SL.

En conclusión, la TP para el SB alto fue 33,3\%, existe una relación funcional significativa y negativa entre el SB y la SL. La dimensión predominante del SB fue la despersonalización y el factor predominante de la SL 
fueron los beneficios económicos. No se han encontrado diferencias significativas al comparar la TP de SB alto, según las variables sociodemográficas y laborales.

\section{REFERENCIAS BIBLIOGRÁFICAS}

1. Da Silva AA, Monteiro Sanchez G, Barbosa Mambrini NS, Ziebell de Oliveira M. Variables de predicción para burnout entre profesionales de enfermería. Rev Psic. 2019; 37(1): 31948.

2. Muñoz SF, Ordoñez-Villota JN, Solarte-Moncayo MN, ValverdeCeron YC, Villarreal-Castro S, Zemanate-Pérez ML. Síndrome de burnout en enfermeros del Hospital Universitario San José. Popayán. Rev Med Risaralda. 2018; 24(1): 34-7.

3. Nantsupawat A, Kunaviktikul W, Nantsupawat R, Wichaikhum $\mathrm{OA}$, Thienthong $\mathrm{H}$, Poghosyan L. Effects of nurse work environment on job dissatisfaction, burnout, intention to leave. Int Nurs Rev. 2017; 64(1): 91-8.

4. Leiter M, Maslach C. Burnout and quality in a sped-up world. J Quality and Participation. 2001; 24: 48-51.

5. Maticorena-Quevedo J, Beas R, Anduaga-Beramendi A, MaytaTristán P. Prevalencia del síndrome de burnout en médicos y enfermeras del Perú, Ensusalud 2014. Rev Perú Med Exp Salud Pública. 2016; 33(2): 241-7.

6. Arauco Pérez GV. Síndrome de Burnout en enfermeros del Hospital Nacional Especializado "Víctor Larco Herrera”, 2007 [Tesis]. Lima: Universidad Nacional Mayor de San Marcos. Facultad de Enfermería; 2008.

7. Vásquez Cucho VK. Síndrome de burnout en anestesiólogos, Hospital Nacional Arzobispo Loayza, 2015 [Tesis]. Lima: Universidad de San Martín de Porres. Facultad de Medicina Humana; 2015.

8. Yslado Méndez RM, Norabuena Figueroa RP, Riveros Quiroz $M$, Sánchez Broncano JD. Programa de intervención psicoeducativo y síndrome burnout en profesionales de salud de hospitales de Casma y Huarmey, Ancash, Perú, 2016. I Congreso Internacional de Psicología y Salud; 2017.

9. Carrillo-García C, Solano-Ruíz MC, Martínez-Roche ME, GómezGarcía Cl. Influencia del género y edad: satisfacción laboral de profesionales sanitarios. Rev Latino-Am Enfermagen. 2013; 21(6): 1314-20.

10. Patlán Pérez J. Efecto del burnout y la sobrecarga en la calidad de vida en el trabajo. Estudios Gerenciales. 2013; 29(129): 445-55.

11. Broncano Vargas YN. Satisfacción laboral y síndrome de burnout en enfermeras del servicio de emergencia y cuidados críticos del Hospital San Bartolomé, 2010. Rev Cient Cien Salud. 2014; 7(2): 53-63.

12. Instituto Nacional de Estadística e Informática. Encuesta nacional de satisfacción de usuarios en salud; 2016. Disponible en: http://portal.susalud.gob.pe/wp-content/ uploads/archivo/encuesta-sat-nac/2016/INFORME_FINAL_ ENSUSALUD_2016.pdf

13. Arbaiza Fermini L. Comportamiento organizacional: bases y fundamentos. 1a ed. Buenos Aires: Cengage Learning; 2010.

14. Maslach C, Jackson SE. MBI-Inventario "Burnout" de Maslach. Síndrome del quemado por estrés laboral asistencial. Madrid: TEA Ediciones; 1997.

15. Solís Chuquiyauri Z, Zamudio Eslava L, Matzumura Kasano J, Gutiérrez Crespo H. Relación entre clima organizacional y síndrome de Burnout en el servicio de emergencia de un
Hospital categoría III-2. Lima, Perú 2015. Horiz Med. 2015; 16(4): 32-8.

16. Bria M, Spânu F, Baban A, Dumitrascu DL. Maslach Burnout Inventory - General Survey: factorial validity and invariance among Romanian healthcare professionals. Burnout Research. 2014; 1(3): 103-11.

17. Palma Carrillo S. Escala de Satisfacción Laboral (SL-SPC). Perú: CARTOLAN EIRL; 2005.

18. Asociación Médica Mundial. Declaración de Helsinki de la AMM: Principios éticos para las investigaciones médicas en seres humanos; 2013. Disponible en: https://www.wma. net/es/policies-post/declaracion-de-helsinki-de-la-ammprincipios-eticos-para-las-investigaciones-medicas-en-sereshumanos/

19. Bonita R, Beaglehole R, Kjellström T. Epidemiología básica. 2a edición. Washington D. C.: Oficina Panamericana de la salud; 2008.

20. Byrne B. Structural Equation Modeling with AMOS: basic concepts, applications, and programming. 2d edition. New York: Routledge Taylor \& Francis Group; 2010.

21. El-Sahili González LF. Burnout: consecuencias y soluciones. México D. F.: Manual Moderno; 2015.

22. Tarcan M, Hikmet N, Schooley B, Top M, Tarcan GY. An analysis of the relationship between burnout, socio-demographic and workplace factors and job satisfaction among emergency department health professionals. Appl Nurs Res. 2017; 34: 407.

23. Espinoza-Lujan C, Lezama-Saavedra N. Síndrome de burnout y factores laborales en profesional de enfermería, Hospital La Caleta-Chimbote, 2010 [Tesis]. Nuevo Chimbote: Universidad Nacional del Santa. Facultad de Enfermería; 2010.

24. Flores Tesillo SY, Ruíz Martínez AO. Burnout en profesionales de la salud y personal administrativo en una unidad médica de primer nivel. Psic Salud. 2018; 28(1): 63-72.

25. Akpinar AT, Taș Y. Acil servis Çalıșanlarının Tükenmișlik ile İș Doyum Düzeyleri Arasındaki iliş̦kiyi Belirlemeye Yönelik Bir Araștırma. Tr J Emerg Med. 2011; 11(4): 161-5.

26. Amaya-Mendoza NM, Santa María-Centurión DL. Síndrome de burnout y satisfacción laboral del profesional de enfermería, Hospital de Apoyo La Caleta, Chimbote-2012 [Tesis]. Nuevo Chimbote: Universidad Nacional del Santa. Facultad de Enfermería; 2012.

27. Díaz Echenique MS, Stimolo MI, Caro NP. Satisfacción laboral y síndrome de desgaste laboral en enfermeros de hospitales públicos Córdoba-Argentina. Med Segur Trab. 2010; 56(218): 22-38.

28. Soto Fuentes PE, Barrios Araya S, Molina Muñoz Y. Síndrome de quemarse por el trabajo y satisfacción laboral como predictores de calidad de la atención de enfermería hospitalaria. Cienc Enferm. 2017; 23(3): 99-111.

29. Tziner A, Rabenu E, Radomski R, Belkin A. Work stress and turnover intentions among hospital physicians: the mediating role of burnout and work satisfaction. Rev Psicol Trab Organ. 2015; 31(3): 207-13.

30. Yang S, Liu D, Liu H, Zhang J, Duan Z. Relationship of work-family conflict, self-reported social support and job satisfaction to burnout syndrome among medical workers in southwest China: a cross-sectional study. PLoS One. 2017; 12(2): e0171679.

31. Gómez-Urquiza JL, Monsalve-Reyes CS, San Luis-Costas C, Fernández-Castillo R, Aguayo-Estremera R, Cañadas-de 
la Fuente GA. Factores de riesgo y niveles de burnout en enfermeras de atención primaria: una revisión sistemática. Aten Primaria. 2017; 49(2): 77-85.

32. Vargas C, Cañadas-de la Fuente GA, Aguayo-Estremera R, Fernández-Castillo R, De la Fuente El. Which occupational risk factors are associated with burnout in nursing? A metaanalytic study. Int J Clin Health Psychol. 2014; 14(1): 28-38.

33. Escriba-Agüir V, Artazcoz L, Pérez-Hoyos S. Efecto del ambiente psicosocial y de la satisfacción laboral en el síndrome de burnout en médicos especialistas. Gac Sanit. 2008; 22(4): 300-8.

34. Contreras Torres F, Espinal L, Pachón AM, González J. Burnout, liderazgo y satisfacción laboral en el personal asistencial de un Hospital de tercer nivel en Bogotá. Divers: Perspect Psicol. 2013; 9(1): 65-80.

35. Gil-Monte PR, Pierre J. El síndrome de quemarse por el trabajo (burnout). En: Moreno-Jiménez O, Garrosa E, editors. Salud laboral. Riesgos laborales psicosociales y bienestar laboral. Madrid: Pirámide; 2015. p 155-175.

36. Joffre-Velázquez VM. Síndrome de burnout en profesionales de la salud en el Hospital General Dr. Carlos Canseco (Tampico, Tamaulipas, México) [Tesis]. Granada: Universidad de Granada. Facultad de Medicina; 2009.

37. Albendín L, Gómez JL, Cañadas-de la Fuente G, Cañadas GR, San Luis-Costas C, Aguayo-Estremera R. Prevalencia bayesiana y niveles de burnout en enfermería de urgencias: una revisión sistemática. Rev Latinoam Psicol. 2016; 48(2): 137-45.

38. Sarmiento Valverde GS. Burnout en el servicio de emergencia de un hospital. Horiz Med. 2019; 19(1): 67-72.

39. Instituto Nacional de Estadística e Informática. Encuesta nacional de satisfacción de usuarios del aseguramiento universal en salud: Profesionales de la salud médicos y enfermeras/os; 2014. Disponible en: https://www.inei. gob.pe/media/MenuRecursivo/publicaciones_digitales/Est/ Lib1192/index.html

40. Superintendencia Nacional de Salud. Resultados definitivos ENSUSALUD; 2016. Disponible en: http://portal.susalud.gob. pe/wp-content/uploads/archivo/encuesta-sat-nac/2016/ PRESENTACION-SUSALUD-2016.pdf

41. Tarcan GY, Tarcan M, Top M. An analysis of relationship between burnout and job satisfaction among emergency health professionals. Total Qual Manag Bus. 2017; 28(11-12): 1339-56.

42. Boria-Reverter S, Crespí-Vallbona M, Mascarilla-Miró 0 . Variables determinantes de la satisfacción laboral en España. Rev Cuadernos de Economía. 2012; 35(97): 9-16.

43. De Simone S, Planta A, Cicotto G. The role of job satisfaction,

work engagement, self-efficacy and agentic capacities on nurses' turnover intention and patient satisfaction. Appl Nurs Res. 2018; 39: 130-40.

44. Suárez RN, Asenjo MBA, Sánchez MD. Job satisfaction among emergency department staff. Australas Emerg Nurs J. 2017; 20(1): 31-6.

45. Robbins SP, Coulter M. Administración. México: Prentice-Hall Hispanoamericana; 2005.

46. Mababu R. Comportamiento humano en el trabajo. Madrid: Ediciones CEF; 2014.

47. Robbins SP. Comportamiento Organizacional. 10a ed. México D. F.: Prentice-Hall Hispanoamericana; 2004.

48. Orgambídez-Ramos A, De Almeida H. Work engagement, social support, and job satisfaction in portuguese nursing staff: a winning combination. Appl Nurs Res. 2017; 36: 37-41.

Fuentes de financiamiento:

Este artículo ha sido financiado por los autores.

Conflictos de interés:

Los autores declaran no tener ningún conflicto de interés.

\section{Correspondencia:}

Rosario Margarita Yslado Méndez

Dirección: Jr. San Martin N ${ }^{\circ} 409$ Huaraz, Ancash.

Teléfono: 949867626

Correo electrónico: rysladom@unasam.edu.pe

Recibido: 13 de mayo de 2019.
Evaluado: 28 de junio de 2019.
Aprobado: 16 de julio de 2019.

(c) La revista. Publicado por Universidad de San Martín de Porres, Perú. (c) Br Licencia de Creative Commons Artículo en acceso abierto bajo términos de Licencia Creative Commons Atribución 4.0 Internacional. (http://creativecommons.org/licenses/by/4.0/)

ORCID iDs

Rosario Margarita Yslado Méndez Roger Pedro Norabuena Figueroa Telmo Pablo Loli Poma

Eva Zarzosa Marquez

Laura Padilla Castro

Irene Pinto Flores

Angelita Rojas Gamboa https://orcid.org/0000-0001-6820-8607 https: / / orcid.org/0000-0003-3731-9843 https: / / orcid.org/0000-0002-6770-9407 https://orcid.org/0000-0002-8041-5227 https://orcid.org/0000-0002-1212-1462 https://orcid.org/0000-0002-3791-4522 https://orcid.org/0000-0003-4911-5645 